\title{
AN OPERATOR BOUND RELATED TO FEYNMAN-KAC FORMULAE
}

\author{
BRIAN JEFFERIES
}

(Communicated by Palle E. T. Jorgensen)

\begin{abstract}
Those Fourier matrix multiplier operators which are convolutions with respect to a matrix valued measure are characterised in terms of an operator bound. As an application, the finite-dimensional distributions of the process associated with Dirac equation are shown to be unbounded on the algebra of cylinder sets.
\end{abstract}

In its traditional incarnation, the Feynman-Kac formula is a means of expressing a perturbation to the heat semigroup in terms of an integral with respect to Wiener measure. It has been useful in proving estimates in quantum physics [Si], and an analogue of the formula is an important tool of quantum field theory [G-J]. Perturbations of the groups of operators associated with certain classes of hyperbolic differential equations can also be represented in terms of integrals with respect to $\sigma$-additive operator valued measures along the lines of the Feynman-Kac formula [I2], [J1]. To establish the existence of $\sigma$-additive operator valued measures associated with particular evolution equations, the following question arises. Suppose that $\Sigma$ is a locally compact abelian group with a given Haar measure $\lambda$. Let $n=1,2, \ldots$ and let $T: L^{2}\left(\Sigma, \mathbb{C}^{n}\right) \rightarrow L^{2}\left(\Sigma, \mathbb{C}^{n}\right)$ be a Fourier matrix multiplier operator acting on the space $L^{2}\left(\Sigma, \mathbb{C}^{n}\right)$ of all ( $\lambda$-equivalence classes of) functions square integrable with respect to $\lambda$ and with values in $\mathbb{C}^{n}$. This means that if the Fourier transform of a function $g \in L^{2}\left(\Sigma, \mathbb{C}^{n}\right)$ is denoted by $\hat{g}$, then there exists a bounded Borel measurable function $\Phi_{T}: \Gamma \rightarrow \mathscr{L}\left(\mathbb{C}^{n}\right)$ from the group $\Gamma$ dual to $\Sigma$ into the space of linear maps $\mathscr{L}\left(\mathbb{C}^{n}\right)$ on $\mathbb{C}^{n}$, such that for every $f \in L^{2}\left(\Sigma, \mathbb{C}^{n}\right)$, the equality $(T f)^{\wedge}(\gamma)=\Phi_{T}(\gamma) \hat{f}(\gamma)$ holds for almost all $\gamma \in \Gamma$. Let $Q$ be the spectral measure acting on $L^{2}\left(\Sigma, \mathbb{C}^{n}\right)$ of multiplication by the characteristic functions of Borel subsets of $\Sigma$.

When is it true that for some $C>0$, the inequality

$$
\left\|\sum_{j=1}^{k} Q\left(g_{j}\right) T Q\left(f_{j}\right)\right\| \leq C\left\|\sum_{j=1}^{k} f_{j} \otimes g_{j}\right\|_{\infty}
$$

holds for all bounded scalar valued Borel measurable functions $f_{j}, g_{j}, j=$

Received by the editors April 2, 1993.

1991 Mathematics Subject Classification. Primary 47A30, 43A25; Secondary 81S40, 35L45.

The author appreciates the hospitality of Macquarie University where the present work was completed. 
$1, \ldots, k$, defined on $\Sigma$ and all $k=1,2, \ldots$ ? Here $u \otimes v$ denotes the function $(u \otimes v)(x, y)=u(x) v(y)$ and the norm $\|\cdot\|_{\infty}$ denotes the $L^{\infty}$-norm with respect to $\lambda \otimes \lambda$ on $\Sigma \times \Sigma$. Another way of stating inequality (1) is that the bilinear map $(f, g) \mapsto Q(g) T Q(f), f, g \in C_{0}(\Sigma)$, is continuous for the topology of bi-equicontinuous convergence. It is not surprising that (1) holds if and only if the operator $T$ is convolution with respect to a matrix-valued measure; see Theorem 1 below, where the result is formulated for an arbitrary separable Hilbert space $H$ in place of $\mathbb{C}^{n}$.

To see what the operator bound (1) has to do with the Feynman-Kac formula, suppose that $\Sigma=\mathbb{R}^{d}$ and let $S$ be a $C_{0}$-semigroup of continuous linear operators acting on $L^{2}\left(\mathbb{R}^{d}, \mathbb{C}^{n}\right)$. If $H$ is the infinitesimal generator of $S$, then $S(t) u_{0}, t \geq 0$, is the solution of the initial-value problem $u^{\prime}(t)=$ $H u(t), u(0)=u_{0}$ in the case that $u_{0}$ belongs to the domain of $H$. For example, with $H=1 / 2 \Delta$ for the Laplacian $\Delta$ acting in $L^{2}\left(\mathbb{R}^{d}, \mathbb{C}\right), S$ is the heat semigroup and the corresponding the initial value problem is the heat equation $\partial_{t} u(x, t)=1 / 2 \Delta u(x, t), u(\cdot, 0)=u_{0}$.

Denote the collection of all functions $\omega:[0, \infty) \rightarrow \mathbb{R}^{d}$ by $\Omega$. Let $t>$ $0, m=1,2, \ldots, 0<t_{1}<\cdots<t_{m}<t$, and suppose that $B_{1}, \ldots, B_{m}$ are Borel subsets of $\mathbb{R}^{d}$. For each subset $E$ of $\Omega$ of the form $E=\left\{\omega \in \Omega: \omega\left(t_{1}\right) \in\right.$ $\left.B_{1}, \ldots, \omega\left(t_{m}\right) \in B_{m}\right\}$, the operator $M_{t}(E) \in \mathscr{L}\left(L^{2}\left(\mathbb{R}^{d}, \mathbb{C}^{n}\right)\right)$ is defined by the formula

$$
M_{t}(E)=S\left(t-t_{m}\right) Q\left(B_{m}\right) S\left(t_{m}-t_{m-1}\right) \cdots Q\left(B_{2}\right) S\left(t_{2}-t_{1}\right) Q\left(B_{1}\right) S\left(t_{1}\right) .
$$

Then as the times $t_{1}, \ldots, t_{m}$, the Borel sets $B_{1}, \ldots, B_{m}$ and $m=1,2, \ldots$ vary, but $t$ is fixed, the sets $E$ form a semialgebra $\mathscr{S}_{t}$ of subsets of $\Omega$ and the expression (2) defines an additive operator valued set function $M_{t}$, defined on $\mathscr{S}_{t}$ and acting on $L^{2}\left(\mathbb{R}^{d}, \mathbb{C}^{n}\right)$. Furthermore, the additivity of the set function $M_{t}$ ensures that it has a unique extension, also denoted by $M_{t}$, to the algebra $\alpha\left(\mathscr{S}_{t}\right)$ of subsets of $\Omega$ generated by $\mathscr{S}_{t}$. The idea of associating an operator valued set function with an arbitray semigroup and a spectral measure is due to I. Kluvánek [K].

Set $X_{s}(\omega)=\omega(s)$ for all $s \geq 0$ and $\omega \in \Omega$, and for a finite ordered subset $J=\left\{t_{1}, \ldots, t_{m}\right\}$ of $(0, t)$, put $X_{J}(\omega)=\left(X_{t_{1}}(\omega), \ldots, X_{t_{m}}(\omega)\right)$. Then $M_{t} \circ X_{J}^{-1}(C)$ is defined to be equal to the operator $M_{t}\left(X_{J}^{-1}(C)\right)$ for any set $C$ which is the finite union of product sets.

The following phenomona may occur.

(i) For some $0<t_{1}<t_{2}<t$, the set function $M_{t} \circ X_{\left\{t_{1}, t_{2}\right\}}{ }^{-1}$ is unbounded on the algebra generated by product sets in $\mathbb{R}^{d}$;

(ii) for all finite $J \subseteq[0, t]$, the set function $M_{t} \circ X_{J}^{-1}$ is bounded on the algebra generated by product sets, but $M_{t}$ is unbounded on the algebra $\alpha\left(\mathscr{S}_{t}\right)$

(iii) the additive set function $M_{t}$ is bounded on the whole algebra $\alpha\left(\mathscr{S}_{t}\right)$.

Briefly, we can say in the language of random processes that the finite-dimensional distributions of $X_{s}, s \geq 0$, with respect to $M_{t}$ are unbounded in case (i), the finite-dimensional distributions of $X_{s}, s \geq 0$, are bounded in case (ii), but $M_{t}$ is unbounded (on $\alpha\left(\mathscr{S}_{t}\right)$ ) and $M_{t}$ is bounded in case (iii). The group $S(t)=e^{i \Delta t / 2}, t \in \mathbb{R}$, of operators is an example of case (i), $S(t)=e^{z \Delta t / 2}, t \geq 0$, 
is an example of case (ii) whenever $z \in \mathbb{C}, \Im(z) \neq 0$, and $\Re(z)>0$, and (iii) occurs if $\Re(z)>0$ and $\Im(z)=0$; see [J2] and the references therein. Only in case (iii) is $M_{t}$ the restriction of a $\sigma$-additive operator valued measure defined on the $\sigma$-algebra $\sigma\left(\mathscr{S}_{t}\right)$ of cylinder sets generated by $\mathscr{S}_{t}$.

In the case that the semigroup $S$ commutes with translations, it follows from the characterisation of operator norm inequality (1) that case (ii) obtains if and only if for each $0 \leq s<t$, the operator $S(s)$ is convolution with a matrix valued measure; see Corollary 1.

Section 1 is devoted to the just mentioned characterisation of operators satisfying the inequality (1). In section 2 , it is noted that the finite-dimensional distributions associated with the Dirac equation in four space-time dimensions are unbounded, although it is known that case (iii) applies to the Dirac equation in two space-time dimensions [I1],[J1]. More generally, a result from the theory of matrix multipliers [B] enables a characterisation of those hyperbolic systems of partial differential equations with constant matrix coefficients where case (ii) applies; these are the hyperbolic systems treated by T. Ichinose [I2], for which the operator valued set functions $M_{t}$ are automatically bounded and define $\sigma$-additive operator valued measures.

\section{AN OPERATOR NORM INEQUALITY}

Throughout this section, $\Sigma$ is a locally compact abelian group with a given Haar measure $\lambda$. The group dual to $\Sigma$ is denoted by $\Gamma$, and its Haar measure is denoted by $\lambda^{\prime}$. The value $\gamma(\sigma)$ of a character $\gamma \in \Gamma$ at $\sigma \in \Sigma$ is written as $\langle\sigma, \gamma\rangle$. The measure $\lambda^{\prime}$ is so normalised that the Fourier-Plancherel formula is valid, that is, for all $f \in L^{1}(\Sigma)$, set $\hat{f}(\gamma)=\int_{\Sigma} f(\sigma) \overline{\langle\sigma, \gamma\rangle} d \lambda(\sigma)$ for all $\gamma \in \Gamma$; then $f(\sigma)=\int_{\Sigma} \hat{f}(\gamma)\langle\sigma, \gamma\rangle d \lambda^{\prime}(\gamma)$ for almost all $\sigma \in \Sigma$ if $f \in L^{1}(\Sigma)$ and $\hat{f} \in L^{1}(\Gamma)$, and $\|f\|_{2}=\|\hat{f}\|_{2}$ if $f \in L^{1}(\Sigma) \cap L^{2}(\Sigma)$.

The Borel $\sigma$-algebra of a locally compact Hausdorff space $X$ is denoted by $\mathscr{B}(X)$. The Banach space of all continuous functions on $X$ vanishing at infinity, with the uniform norm, is denoted by $C_{0}(X)$. The variation of a Borel measure $\mu: \mathscr{B}(X) \rightarrow \mathbb{C}$ is denoted by $|\mu|$.

Let $(\Lambda, \mathscr{T}, \mu)$ be a measure space. Suppose that $H$ is a separable Hilbert space with inner product $(\cdot, \cdot)$, antilinear in the second variable, and norm $\|\cdot\|_{H}$. Let $L^{2}(\mu, H)$ be the Hilbert space of ( $\mu$-equivalence classes of) strongly $\mu$-measurable functions $f: \Lambda \rightarrow H$ such that $\left.\|f\|_{2} \equiv\left(\int_{\Lambda}\|f(\xi)\|_{H}^{2}\right) d \mu(\xi)\right)^{1 / 2}$ is finite. Here "strongly $\mu$-measurable" means the limit $\mu$-a.e. of countably valued $H$-valued $\mathscr{T}$-measurable functions. Because $H$ is assumed to be separable, it is enough to assume that the function $\xi \mapsto(f(\xi), h), \xi \in \Lambda$, is $\mu$-measurable for each $h \in H$ [D-U, II.1.2], that is, $f$ is scalarly measurable. The inner product of $L^{2}(\mu, H)$ is defined by $(f, g)=\int_{\Lambda}(f(\sigma), g(\sigma)) d \mu(\sigma), f, g \in L^{2}(\mu, H)$. For the applications of section two, $H$ is $\mathbb{C}^{n}$. The space of continuous linear operators on $H$ is denoted by $\mathscr{L}(H)$; it is equipped with the topology of strong operator convergence.

We now extend some notions from commutative harmonic analysis to the vector valued setting. The Fourier-Plancherel formula also applies to $L^{2}(\Sigma, H)$, because $L^{2}(\Sigma, H)$ is isomorphic to the Hilbert space tensor product of $L^{2}(\Sigma)$ and $H$ so that the Fourier transform ${ }^{\wedge}: L^{2}(\Sigma, H) \rightarrow L^{2}(\Gamma, H)$ is an isometry.

A continuous linear operator $T: L^{2}(\Sigma, H) \rightarrow L^{2}(\Sigma, H)$ is called a Fourier 
multiplier operator if there exists a function $\Phi: \Gamma \rightarrow \mathscr{L}(H)$, such that for each $h \in H$, the function $\Phi h: \gamma \mapsto \Phi(\gamma) h, \gamma \in \Gamma$, is strongly $\lambda^{\prime}$-measurable, $\Phi$ is $\lambda^{\prime}$-essentially bounded in the operator norm of $\mathscr{L}(H)$ and for each $f \in$ $L^{2}(\Sigma, H)$, the equality $(T f)(\gamma)=\Phi(\gamma) \hat{f}(\gamma)$ holds for $\lambda^{\prime}$-almost all $\gamma \in \Gamma$. The separability of $H$ ensures that the function $\gamma \mapsto\|\Phi(\gamma)\|, \gamma \in \Gamma$, is measurable and that $T: L^{2}(\Sigma, H) \rightarrow L^{2}(\Sigma, H)$ is a Fourier multiplier operator if and only if it commutes with each unitary operator $U_{\tau}, \tau \in \Sigma$, sending $f \in L^{2}(\Sigma, H)$ to the function $\sigma \mapsto f(\sigma \tau), \sigma \in \Sigma$. In the case that $H=\mathbb{C}^{n}$, the values of $\Phi$ may be viewed as $(n \times n)$-matrices relative to the standard basis of $\mathbb{R}^{n}$ and for $f \in L^{2}\left(\Sigma, \mathbb{C}^{n}\right),(\Phi(\gamma) \hat{f}(\gamma))_{j}=\sum_{k=1}^{n} \Phi(\gamma)_{j k} \hat{f}(\gamma)_{k}$, for each $j=1, \ldots, n$.

Lemma 1. For any function $\Phi: \Gamma \rightarrow \mathscr{L}(H)$, such that for each $h \in H$, the function $\Phi h: \gamma \mapsto \Phi(\gamma) h, \gamma \in \Gamma$, is strongly $\lambda^{\prime}$-measurable and $\Phi$ is $\lambda^{\prime}$-essentially bounded in the operator norm of $\mathscr{L}(H)$, there exists a unique bounded linear operator $T_{\Phi}: L^{2}(\Sigma, H) \rightarrow L^{2}(\Sigma, H)$ such that for each $f \in$ $L^{2}(\Sigma, H)$, the equality $\left(T_{\Phi} f\right)^{\prime}(\gamma)=\Phi(\gamma) \hat{f}(\gamma)$ holds for almost all $\gamma \in \Gamma$. The operator norm of $T_{\Phi}$ is equal to $\operatorname{ess.sup}_{\gamma \in \Gamma}\|\Phi(\gamma)\|$.

Proof. The function $\gamma \mapsto \Phi(\gamma) g(\gamma), \gamma \in \Gamma$, is strongly measurable for each $g \in L^{2}(\Gamma, H)$, because if $s_{n}, n=1,2, \ldots$, are countably $H$-valued measurable functions converging off a $\lambda^{\prime}$-null set $N$ to $g$, then for all $\gamma \in \Sigma \backslash N$, $\lim _{n \rightarrow \infty} \Phi(\gamma) s_{n}(\gamma)=\Phi(\gamma) g(\gamma)$, because $\Phi(\gamma)$ is a continuous linear operator on $H$; moreover, each of the functions $\gamma \mapsto \Phi(\gamma) s_{n}(\gamma), \gamma \in \Gamma, n=1,2, \ldots$, is strongly $\lambda^{\prime}$-measurable and so is their pointwise limit.

Let $\|\Phi\|_{\infty}$ denote the essential supremum $\operatorname{ess.sup}_{\gamma \in \Gamma}\|\Phi(\gamma)\|$. If $f \in$ $L^{2}(\Sigma, H)$, then $\|\Phi \hat{f}\|_{2} \leq\|\Phi\|_{\infty}\|\hat{f}\|_{2}$. By the Plancherel theorem, the equality $\left(T_{\Phi} f\right)(\gamma)=\Phi(\gamma) \hat{f}(\gamma)$ for almost all $\gamma \in \Gamma$ defines an element $T_{\Phi} f$ of $L^{2}(\Sigma, H)$ such that $\left\|T_{\Phi} f\right\|_{2}=\|\Phi \hat{f}\|_{2} \leq\|\Phi\|_{\infty}\|\hat{f}\|_{2}=\|\Phi\|_{\infty}\|f\|_{2}$, so the mapping $f \mapsto T_{\Phi} f, f \in L^{2}(\Sigma, H)$, is a bounded linear operator $T_{\Phi}$ on $L^{2}(\Sigma, H)$ with norm at most $\|\Phi\|_{\infty}$.

To see that the norm $\left\|T_{\Phi}\right\|$ of $T_{\Phi}$ is actually equal to the essential supremum $\|\Phi\|_{\infty}$, let $u_{j}, j=1,2, \ldots$, be a countable dense subset of the closed unit ball of $H$. For all $u, v \in H$, set $\Phi_{u, v}(\gamma)=(\Phi(\gamma) u, v)$ for every $\gamma \in \Gamma$. Let $\epsilon>0$, and for each $j, k=1,2, \ldots$, set $A_{j, k}=\left\{\gamma \in \Gamma:\left|\Phi_{u_{j}, v_{k}}(\gamma)\right|>\right.$ $\left.\|\Phi\|_{\infty}-\epsilon\right\}$. If $\xi \in \cap_{j, k} A_{j, k}^{c}$, then $\|\Phi(\xi)\| \leq\|\Phi\|_{\infty}-\epsilon$, because $\Phi(\xi)$ is a continuous linear operator on $H$. By the definition of $\|\Phi\|_{\infty}$, the set $\cap_{j, k} A_{j, k}^{c}$ cannot be a set of full $\lambda^{\prime}$-measure. Consequently, there exists $j, k=1,2, \ldots$ such that the set $A_{j, k}$ is not $\lambda^{\prime}$-null, so $\left\|\Phi_{u_{j}, v_{k}}\right\|_{\infty}>\|\Phi\|_{\infty}-\epsilon$. The equality $\sup _{\|u\|,\|v\| \leq 1}\left\|\Phi_{u, v}\right\|_{\infty}=\|\Phi\|_{\infty}$ now follows, because for all $u, v \in H$ with $\|u\|,\|v\| \leq 1,\left\|\Phi_{u, v}\right\|_{\infty} \leq\|\Phi\|_{\infty}$. For each $u, v \in H$, define $\left(T_{\Phi} u, v\right)$ : $L^{2}(\Sigma) \rightarrow L^{2}(\Sigma)$ by

$$
\left(\left(T_{\Phi} u, v\right) f\right)(\sigma)=\left(T_{\Phi}(u f)(\sigma), v\right), \quad f \in L^{2}(\Sigma) .
$$

From the scalar case, $\left\|T_{\Phi}\right\|=\sup _{\|u\|,\|v\| \leq 1}\left\|\left(T_{\Phi} u, v\right)\right\|=\sup _{\|u\|,\|v\| \leq 1}\left\|\Phi_{u, v}\right\|_{\infty}$ $=\|\Phi\|_{\infty}$, so the equality $\left\|T_{\Phi}\right\|=\|\Phi\|_{\infty}$ is valid.

Of particular interest is the following special class of Fourier multiplier operators on $L^{2}(\Sigma, H)$. Suppose that $T: L^{2}(\Sigma, H) \rightarrow L^{2}(\Sigma, H)$ is a bounded linear operator for which there exists a regular operator valued measure $\mu$ : 
$\mathscr{B}(\Sigma) \rightarrow \mathscr{L}(H)$, such that for every $u, v \in H$ and $f \in L^{2}(\Sigma)$, the equality $(T(u f)(\sigma), v)=\int_{\Sigma} f\left(\sigma \tau^{-1}\right) d(\mu u, v)(\tau)$ holds for $\lambda$-almost all $\sigma \in \Sigma$. Here $(\mu u, v)$ is the scalar measure $(\mu u, v): A \mapsto(\mu(A) u, v), A \in \mathscr{B}(\Sigma)$, and the regularity of $\mu$ means that for every $u \in H, A \in \mathscr{B}(\Sigma)$, and $\epsilon>0$, there exists a compact subset $K$ of $A$ such that $\|\mu(B) u\|<\epsilon$ for all $B \subseteq A \backslash K$. A reference for integration with respect to vector measures is [D-U]. The set of all finite linear combinations of $H$-valued functions $u f$ is dense in $L^{2}(\Sigma, H)$, so $\mu$ uniquely determines $T$. The operator $T$ is said to be the operator of convolution with respect to $\mu$.

For any regular operator valued measure $\mu: \mathscr{B}(\Sigma) \rightarrow \mathscr{L}(H)$, the continuous operator valued function $\Phi: \Gamma \rightarrow \mathscr{L}(H)$ defined by $\Phi(\gamma)=\int_{\Sigma} \overline{\langle\sigma, \gamma\rangle} d \mu(\sigma)$ is called the Fourier-Stieltjes transform of $\mu$. The total variation $V(\mu)$ of a measure $\mu: \mathscr{B}(\Sigma) \rightarrow \mathscr{L}(H)$ is the (possibly infinite) supremum of the numbers $\sum_{j=1}^{n}\left\|\mu\left(A_{j}\right)\right\|$ over all partitions $A_{j}, j=1, \ldots, n, n=1,2, \ldots$, of $\Sigma$ by Borel sets. If $V(\mu)<\infty$, then $\mu$ is said to have finite variation. For a finitedimensional Hilbert space $H$, all $\mathscr{L}(H)$-valued measures have finite variation, but if $H$ is, say, $L^{2}([0,1])$, then the spectral measure of multiplication by characteristic functions has infinite variation.

The next proposition ensures that any regular $\mathscr{L}(H)$-valued measure $\mu$ uniquely defines a bounded linear operator on $L^{2}(\Sigma, H)$ of convolution with respect to $\mu$.

Proposition 1. Suppose that $T: L^{2}(\Sigma, H) \rightarrow L^{2}(\Sigma, H)$ is the operator of convolution with respect to a regular operator valued measure $\mu: \mathscr{B}(\Sigma) \rightarrow \mathscr{L}(H)$. Then $T$ is the Fourier multiplier operator $T_{\Phi}$ for the Fourier-Stieltjes transform $\Phi$ of $\mu$. Conversely, if $\Phi$ is the Fourier-Stieltjes transform of a regular operator valued measure $\mu: \mathscr{B}(\Sigma) \rightarrow \mathscr{L}(H)$, then $T_{\Phi}$ is the operator of convolution with respect to $\mu$.

Proof. Let $u, v \in H$ and $f \in L^{2}(\Sigma)$. Suppose first that $T$ is the operator convolution with $\mu$. The scalar measure $B \mapsto(\mu(B) u, v), B \in \mathscr{B}(\Sigma)$, is denoted by $(\mu u, v)$. Then $(T(u f), v)=f *(\mu u, v)$, so for almost all $\gamma \in \Gamma,(T(u f), v)^{\wedge}(\gamma)=\left([T(u f)]^{\wedge}(\gamma), v\right)=\Phi_{u, v}(\gamma) \hat{f}(\gamma)$. Here $\Phi_{u, v}$ is the Fourier-Stieltjes transform of the scalar measure $(\mu u, v)$, so for every $\gamma \in \Gamma, \Phi_{u, v}(\gamma)=(\Phi(\gamma) u, v)$ for the Fourier-Stieltjes transform $\Phi$ of $\mu$. The equality $T(u f)=T_{\Phi}(u f)$ therefore holds for all $u \in H$ and $f \in L^{2}(\Sigma)$, so equality holds for the closure in $L^{2}(\Sigma, H)$ of linear combinations of all such functions $u f$, namely, on the whole of $L^{2}(\Sigma, H)$.

Conversely, suppose that $\Phi$ is the Fourier-Stieltjes transform of $\mu$ and let $u, v \in H$ and $f \in L^{2}(\Sigma)$. According to Lemma 1 , the $\mathscr{L}(H)$-valued function $\Phi$ defines the bounded linear operator $T_{\Phi}: L^{2}(\Sigma, H) \rightarrow L^{2}(\Sigma, H)$. Then for almost all $\gamma \in \Gamma$,

$$
\left(\left[T_{\Phi}(u f)\right]^{\wedge}(\gamma), v\right)=\left(T_{\Phi}(u f), v\right)^{\wedge}(\gamma)=\Phi_{u, v}(\gamma) \hat{f}(\gamma)=(f *(\mu u, v))^{\wedge} .
$$

It follows that $T_{\Phi}$ is convolution with $\mu$.

The following are examples of continuous linear maps $T: L^{2}(\Sigma, H) \rightarrow$ $L^{2}(\Sigma, H)$, each of which is the operator of convolution with respect to an operator valued measure with infinite variation. 
Example 1. (i) Set $H=L^{2}(\mathbb{R})$. Then $L^{2}(\mathbb{R}, H)$ can be identified with $L^{2}\left(\mathbb{R}^{2}\right)$ by identifying the $H$-valued function $x \mapsto g(x) h, x \in \mathbb{R}$ with $(x, y) \mapsto$ $g(x) h(y), x, y \in \mathbb{R}^{2}$. Let $Q: \mathscr{B}(\mathbb{R}) \rightarrow \mathscr{L}(H)$ be defined by $Q(B) f=\chi_{B} f$, for every $B \in \mathscr{B}(\mathbb{R})$ and every $f \in L^{2}(\mathbb{R}, H)$. Then the operator $T: L^{2}(\mathbb{R} ; H)$ $\rightarrow L^{2}(\mathbb{R}, H)$ of convolution with $Q$ corresponds to the isometry $f \mapsto f \circ u, f \in$ $L^{2}\left(\mathbb{R}^{2}\right)$ with

$$
u(x, y)=(x-y, y), \quad x, y \in \mathbb{R} .
$$

The measure $Q$ has infinite variation.

(ii) The following example is more interesting. Let $F_{t}(x)=\frac{1}{i \pi} \operatorname{sgn} x \cdot e^{-|x| t}$ for all $x \in \mathbb{R}$ and $t \geq 0$. Denote by $\widehat{F}_{t}(D)$ the operator of convolution with $F_{t}$ on $L^{2}(\mathbb{R})$ for each $t>0$. The notation is motivated by considering the Fourier transform

$$
\left(\widehat{F}_{t}(D) f\right)(\xi)=\widehat{F}_{t}(\xi) \hat{f}(\xi)
$$

for $f \in L^{2}(\mathbb{R})$ and almost all $\xi \in \mathbb{R}$. The operator of multiplication of the Fourier transform by $\xi$ corresponds to $D=\frac{1}{i} \frac{d}{d x}$, so $\widehat{F}_{t}(D)$ is actually the operator obtained from the bounded function $\widehat{F}_{t}$ by the functional calculus for the selfadjoint operator $D$. The function $t \mapsto \widehat{F}_{t}(D), t \geq 0$, is integrable in $\mathscr{L}(H)$, so let

$$
\mu(A)=\int_{A \cap[0, \infty)} \widehat{F}_{t}(D) d t \text { for all } A \in \mathscr{B}(\mathbb{R}) .
$$

The Fourier-Stieltjes transform $\Phi$ of $\mu$ is the bounded operator valued function $\xi \mapsto \int_{0}^{\infty} e^{-i \xi t} \widehat{F}_{t}(D) d t, \xi \in \mathbb{R}$, and according to Proposition 1 , the Fourier multiplier operator $T_{\Phi}$ is the operator of convolution with respect to $\mu$. The measure $\mu$ has infinite variation.

Note that $\mu([0, \infty))$ is the Hilbert transform on $L^{2}(\mathbb{R})$. The details concerning this example, and others constructed from singular integral operators on $L^{2}(\mathbb{R})$, may be gleaned from [J-O].

The next result gives a characterisation of Fourier multiplier operators which are convolutions with respect to an $\mathscr{L}(H)$-valued measure.

Theorem 1. Let $\Sigma$ be a locally compact abelian group, $H$ a separable Hilbert space, and suppose that $T: L^{2}(\Sigma, H) \rightarrow L^{2}(\Sigma, H)$ is a Fourier multiplier operator. Then $T$ is the operator of convolution with respect to a regular operator-valued measure $\mu: \mathscr{B}(\Sigma) \rightarrow \mathscr{L}(H)$ if and only if there exists a constant $C>0$ such that for every $\phi, \psi \in L^{2}(\Sigma)$ and $u, v \in H$,

$$
\left|\sum_{j=1}^{k}\left(T\left(f_{j} \phi u\right), \overline{g_{j}} \psi v\right)\right| \leq C\|u\|_{H}\|v\|_{H}\|\phi\|_{2}\|\psi\|_{2}\left\|\sum_{j=1}^{k} f_{j} \otimes g_{j}\right\|_{\infty}
$$

for all $f_{j}, g_{j} \in L^{\infty}(\Sigma), j=1, \ldots, k$, and $k=1,2, \ldots$

Proof. Suppose that $T$ is convolution with respect to an $\mathscr{L}(H)$-valued measure $\mu$. Let $\phi, \psi \in L^{2}(\Sigma), u, v \in H$, and let $\alpha=\phi u, \beta=\psi v$ be elements of 
$L^{2}(\Sigma, H)$. Then

$$
\begin{aligned}
\mid \sum_{j=1}^{k} & \left(T\left(f_{j} \alpha\right), \overline{g_{j}} \beta\right) \mid \\
& =\left|\sum_{j=1}^{k} \int_{\Sigma}\left[\int_{\Sigma}\left(f_{j} \phi\right)\left(\sigma \tau^{-1}\right) d(\mu u, v)(\tau)\right]\left(g_{j} \bar{\psi}\right)(\sigma) d \lambda(\sigma)\right| \\
& =\left|\sum_{j=1}^{k} \int_{\Sigma}\left[\int_{\Sigma}\left(f_{j} \phi\right)\left(\sigma \tau^{-1}\right)\left(g_{j} \bar{\psi}\right)(\sigma) d \lambda(\sigma)\right] d(\mu u, v)(\tau)\right| \\
& \leq\left\|\sum_{j=1}^{k} f_{j} \otimes g_{j}\right\|_{\infty} \int_{\Sigma}\left[\int_{\Sigma}\left|\phi\left(\sigma \tau^{-1}\right) \| \psi(\sigma)\right| d \lambda(\sigma)\right] d|(\mu u, v)|(\tau) \\
& \leq\|\mu\| .\|u\|_{H}\|v\|_{H}\||\phi| *|\psi|\|_{\infty}\left\|\sum_{j=1}^{k} f_{j} \otimes g_{j}\right\|_{\infty} \\
& \leq\|\mu\| .\|u\|_{H}\|v\|_{H}\|\phi\|_{2}\|\psi\|_{2}\left\|\sum_{j=1}^{k} f_{j} \otimes g_{j}\right\|_{\infty}
\end{aligned}
$$

by the Fubini-Tonelli theorem. Here the semivariation [D-U, I.1.11] $\|\mu\|$ is defined by the formula

$$
\|\mu\|=\sup \left\{|(\mu p, q)|(\Sigma): p, q \in H,\|p\|_{H} \leq 1,\|q\|_{H} \leq 1\right\}
$$

Now suppose that (3) holds and that $T$ is a Fourier multiplier operator. Let $\phi, \psi \in L^{2}(\Sigma), u, v \in H$, and set $\alpha=\phi u, \beta=\psi v$. Then there exists an essentially bounded operator valued function $\Phi: \Sigma \rightarrow \mathscr{L}(H)$ such that $T=T_{\Phi}$.

The collection of all complex functions $\sum_{j=1}^{k} f_{j} \otimes g_{j}, f_{j}, g_{j} \in C_{0}(\Sigma), j=$ $1, \ldots, k, k=1,2, \ldots$, is dense in $C_{0}(\Sigma \times \Sigma)$ by the Stone-Weierstrass theorem. The inequality (3) therefore shows that there exists a unique continuous linear map $\Psi_{\alpha, \beta}: C_{0}(\Sigma \times \Sigma) \rightarrow \mathbb{C}$ such that $\Psi_{\alpha, \beta}(f \otimes g)=(T(f \alpha), \bar{g} \beta)$ for all $f, g \in C_{0}(\Sigma)$ and $\left\|\Psi_{\alpha, \beta}\right\| \leq C\|\alpha\|_{2}\|\beta\|_{2}$. The Riesz representation theorem ensures that there exists a unique regular Borel measure $\nu_{\alpha, \beta}: \mathscr{B}(\Sigma \times \Sigma) \rightarrow \mathbb{C}$ such that $\Psi_{\alpha, \beta}(\Lambda)=\int_{\Sigma \times \Sigma} \Lambda(\sigma, \tau) d \nu_{\alpha, \beta}(\sigma, \tau)$ for all $\Lambda \in C_{0}(\Sigma \times \Sigma)$. Another appeal to the Riesz representation theorem shows that there exists a unique regular Borel measure $\mu_{\alpha, \beta}: \mathscr{B}(\Sigma) \rightarrow \mathbb{C}$ such that

$$
\int_{\Sigma} \Lambda(\sigma) d \mu_{\alpha, \beta}(\sigma)=\int_{\Sigma \times \Sigma} \Lambda\left(\tau \sigma^{-1}\right) d \nu_{\alpha, \beta}(\sigma, \tau)
$$

for all $\Lambda \in C_{0}(\Sigma)$ and whose total variation $\left|\mu_{\alpha, \beta}\right|(\Sigma)$ is bounded by $C\|\alpha\|_{2}\|\beta\|_{2}$. Let $w: \Sigma \rightarrow \mathbb{C}$ be a function such that $\hat{w} \in L^{1}(\Gamma)$. Then $w \in C_{0}(\Sigma)$ and by 
Fubini's theorem and the Plancherel formula,

$$
\begin{aligned}
\int_{\Sigma} w & (\sigma) d \mu_{\alpha, \beta}(\sigma)=\int_{\Sigma \times \Sigma} w\left(\tau \sigma^{-1}\right) d \nu_{\alpha, \beta}(\sigma, \tau) \\
= & \int_{\Sigma \times \Sigma}\left[\int_{\Gamma}\left\langle\tau \sigma^{-1}, \gamma\right\rangle \hat{w}(\gamma) d \lambda^{\prime}(\gamma)\right] d \nu_{\alpha, \beta}(\sigma, \tau) \\
& =\int_{\Gamma}\left[\int_{\Sigma \times \Sigma}\left\langle\tau \sigma^{-1}, \gamma\right\rangle d \nu_{\alpha, \beta}(\sigma, \tau)\right] \hat{w}(\gamma) d \lambda^{\prime}(\gamma) \\
& =\int_{\Gamma}\left[\int_{\Sigma \times \Sigma}\langle\tau, \gamma\rangle \overline{\langle\sigma, \gamma\rangle} d \nu_{\alpha, \beta}(\sigma, \tau)\right] \hat{w}(\gamma) d \lambda^{\prime}(\gamma) \\
& =\int_{\Gamma}(T(\bar{\gamma} \alpha), \bar{\gamma} \beta) \hat{w}(\gamma) d \lambda^{\prime}(\gamma) \\
& =\int_{\Gamma}\left[\int_{\Gamma}(\Phi(\xi)(\bar{\gamma} \alpha)(\xi),(\bar{\gamma} \beta)(\xi)) d \lambda^{\prime}(\xi)\right] \hat{w}(\gamma) d \lambda^{\prime}(\gamma) \\
& =\int_{\Gamma}\left[\int_{\Gamma}(\Phi(\xi) \hat{\alpha}(\xi \gamma), \hat{\beta}(\xi \gamma)) \hat{w}(\gamma) d \lambda^{\prime}(\gamma)\right] d \lambda^{\prime}(\xi) \\
& =\int_{\Gamma}\left[\int_{\Gamma}\left(\Phi(\xi) \hat{\alpha}\left(\xi \gamma^{-1}\right), \hat{\beta}\left(\xi \gamma^{-1}\right)\right) \hat{w}\left(\gamma^{-1}\right) d \lambda^{\prime}(\gamma)\right] d \lambda^{\prime}(\xi) .
\end{aligned}
$$

Hence, the absolute value of the last integral is bounded by $C\|w\|_{\infty}\|\alpha\|_{2}\|\beta\|_{2}$.

Now let $\mathscr{U}$ denote the family of neighbourhoods of the identity in $\Gamma$ directed by inclusion. If $f_{U} \geq 0$ vanishes off $U \in \mathscr{U}$ and satisfies $\int_{\Sigma} f_{U} d \lambda=1$, then $\left\{f_{U}\right\}_{U \in \mathscr{U}}$ is an approximate unit for $L^{1}(\Gamma)$. For each $U \in \mathscr{U}$, let $g_{U} \in L^{2}(\Sigma)$ be the essentially unique function satisfying $\hat{g}_{U}=f_{U}^{1 / 2}$. If we set $\phi=g_{U}$ and $\psi=R \overline{g_{U}}$, then $\|\alpha\|_{2}=\|u\|_{H}$ and $\|\beta\|_{2}=\|v\|_{H}$ and the integral above becomes $\int_{\Gamma}(\Phi(\xi) u, v) f_{U^{*}}(R \hat{w})(\xi) d \lambda^{\prime}(\xi)$, where $R$ is the inversion operator given by $R p(\gamma)=p\left(\gamma^{-1}\right)$, for all measurable functions $p$ and $\gamma \in \Gamma$.

Because $\lim _{U \in \mathscr{U}} f_{U} * \hat{w}=\hat{w}$ in $L^{1}(\Gamma)$, it follows that

$$
\left|\int_{\Gamma}(\Phi(\xi) u, v) \hat{w}\left(\xi^{-1}\right) d \lambda^{\prime}(\xi)\right| \leq C\|w\|_{\infty}\|u\|_{2}\|v\|_{2} .
$$

Moreover, the measures $\mu_{\alpha, \beta}$ converge in the total variation norm to a measure $\mu_{u, v}$ satisfying $\left|\mu_{u, v}\right|(\Sigma) \leq C\|u\|_{H}\|v\|_{H}$. Consequently, there exists an operator valued measure $\mu: \mathscr{B}(\Sigma) \rightarrow \mathscr{L}(H)$ such that $(\mu u, v)=\mu_{u, v}$ for all $u, v \in H$. The $\sigma$-additivity of $\mu$ in the strong operator topology of $\mathscr{L}(H)$ follows from the Orlicz-Pettis theorem [D-U, I.4.4]. The regularity of $\mu$ follows from the regularity of $\mu_{u, v}$ for all $u, v \in H$ and [D-U, I.2.4].

The identity $\int_{\Gamma} \Phi\left(\xi^{-1}\right) \hat{w}(\xi) d \lambda^{\prime}(\xi)=\int_{\Sigma} w(\sigma) d \mu(\sigma)$ is valid for all $w: \Sigma \rightarrow \mathbb{C}$ such that $\hat{w} \in L^{1}(\Gamma)$, so by the Plancherel formula, $\int_{\Gamma} \Phi\left(\xi^{-1}\right) u(\xi) d \lambda^{\prime}(\xi)=$ $\int_{\Sigma} \check{u}(\sigma) d \mu(\sigma)$ for the inverse Fourier transform $\check{u}$ of a function $u \in L^{1}(\Gamma)$. By Fubini's theorem, it follows that $\Phi$ is the Fourier-Stieltjes transform of the regular operator valued measure $\mu: \mathscr{B}(\Sigma) \rightarrow \mathscr{L}(H)$. An appeal to Proposition 1 establishes that $T$ is convolution with $\mu$.

Corollary 1. Let $\Sigma$ be a locally compact abelian group, $H$ a separable Hilbert space, and $\mu: \mathscr{B}(\Sigma) \rightarrow \mathscr{L}(H)$ a regular $\mathscr{L}(H)$-valued measure with finite variation. Let $Q$ be the spectral measure of multiplication by Borel subsets of 
$\Sigma$, acting on $L^{2}(\Sigma, H)$. Suppose that $T: L^{2}(\Sigma, H) \rightarrow L^{2}(\Sigma, H)$ is the operator of convolution with respect to $\mu$. Then there exists a constant $C>0$ such that

$$
\left\|\sum_{j=1}^{k} Q\left(g_{j}\right) T Q\left(f_{j}\right)\right\| \leq C\left\|\sum_{j=1}^{k} f_{j} \otimes g_{j}\right\|_{\infty}
$$

for all $f_{j}, g_{j} \in L^{\infty}(\Sigma), j=1, \ldots, k$, and $k=1,2, \ldots$.

Proof. The proof is along the lines of the first part of the proof of Theorem 1. Suppose that $\xi_{j}, j=1 \ldots, k$, are the characteristic functions of pairwise disjoint Borel subsets of $\Sigma$; similarly for $\zeta_{j}, j=1, \ldots, k$. Then for $u_{j}, v_{j} \in$ $H, j=1, \ldots, k, \sum_{j=1}^{k} u_{j} \xi_{j}$ and $\sum_{j=1}^{k} v_{j} \zeta_{j}$ are $H$-valued simple functions. Some of these vectors may be zero. Then

$$
\begin{aligned}
& \left|\sum_{l=1}^{m} \sum_{i, j=1}^{k}\left(T\left(u_{i} f_{l} \xi_{i}\right), v_{j} \bar{g}_{l} \zeta_{j}\right)\right| \\
& \quad=\left|\sum_{l=1}^{m} \sum_{i, j=1}^{k} \int_{\Sigma}\left[\int_{\Sigma}\left(f_{l} \xi_{i}\right)\left(\sigma \tau^{-1}\right)\left(g_{l} \zeta_{j}\right)(\sigma) d \lambda(\sigma)\right] d\left(\mu u_{i}, v_{j}\right)(\tau)\right| \\
& \quad \leq\left\|\sum_{l=1}^{m} f_{l} \otimes g_{l}\right\|_{\infty} \times \sum_{i, j=1}^{k} \int_{\Sigma}\left[\int_{\Sigma}\left|\xi_{i}\left(\sigma \tau^{-1}\right) \| \zeta_{j}(\sigma)\right| d \lambda(\sigma)\right] d\left|\left(\mu u_{i}, v_{j}\right)\right|(\tau) \\
& \quad \leq V(\mu)\left\|\sum_{l=1}^{m} f_{l} \otimes g_{l}\right\|_{\infty} \sup _{\tau} \sum_{i, j=1}^{k} \int_{\Sigma}\left|\xi_{i}\left(\sigma \tau^{-1}\right)\right| \cdot\left\|u_{i}\right\|_{H}\left|\zeta_{j}(\sigma)\right| \cdot\left\|v_{j}\right\|_{H} d \lambda(\sigma) \\
& \quad \leq V(\mu)\left\|\sum_{i=1}^{k} u_{i} \xi_{i}\right\|_{2}\left\|\sum_{j=1}^{k} v_{j} \zeta_{j}\right\|\left\|\sum_{2}^{m} f_{l=1}^{m} \otimes g_{l}\right\|_{\infty} .
\end{aligned}
$$

Simple functions are dense in $L^{2}(\Sigma, H)$, so the required estimate follows by continuity.

Remarks. (i) Clearly, inequality (4) implies (3). Furthermore, if $H$ is finite dimensional, then (4) holds for any $\mathscr{L}(H)$-valued measure, because all such measures automatically have finite variation.

(ii) Inequality (4) does not even imply that for each $h \in H$, the $H$-valued measure $\mu h$ has finite variation. For example, if $Q$ is the spectral measure and $T$ is the operator of Example $1(\mathrm{i})$, then $\sum_{j=1}^{k}\left(Q\left(g_{j}\right) T Q\left(f_{j}\right) \phi\right)(x, y)=$ $\sum_{j=1}^{k} f_{j}(x-y) g_{j}(x) \phi(x-y, y)$, for all $\phi \in L^{2}\left(\mathbb{R}^{2}\right)$, so (4) is clearly satisfied. But, as mentioned earlier, $Q h$ has finite variation in $H$ only if $h$ is the zero vector.

\section{EVOLUTION EQUATIONS}

The Dirac operator is defined in $L^{2}\left(\mathbb{R}^{3}, \mathbb{C}^{4}\right)$ by means of the differential expression

$$
D=c \sum_{j=1}^{3} \alpha_{j} p_{j}+\alpha_{4} m c^{2},
$$


where $c>0$ is the velocity of light, $m>0$ is the mass of the particle, $p_{j}=$ $\frac{\hbar}{i} \frac{\partial}{\partial x_{j}}$, and

$$
\alpha_{j}=\left(\begin{array}{cc}
0 & \sigma_{j} \\
\sigma_{j} & 0
\end{array}\right), \quad j=1,2,3, \quad \alpha_{4}=\left(\begin{array}{cc}
\sigma_{0} & 0 \\
0 & -\sigma_{0}
\end{array}\right) .
$$

Here $\sigma_{1}, \sigma_{2}, \sigma_{2}$ are the Pauli matrices

$$
\sigma_{1}=\left(\begin{array}{cc}
0 & 1 \\
1 & 0
\end{array}\right), \quad \sigma_{2}=\left(\begin{array}{cc}
0 & -i \\
i & 0
\end{array}\right), \quad \sigma_{3}=\left(\begin{array}{cc}
1 & 0 \\
0 & -1
\end{array}\right)
$$

and $\sigma_{0}=\left(\begin{array}{ll}1 & 0 \\ 0 & 1\end{array}\right)$ is the $2 \times 2$ identity matrix.

Then $D$ defines a selfadjoint operator, and so, a unitary group $S(t)=$ $e^{i D t}, t \in \mathbb{R}$, of operators acting on $L^{2}\left(\mathbb{R}^{3}, \mathbb{C}^{4}\right)$. The question arises as to whether or not the finite-dimensional distributions of the process defined by formula (2) are bounded.

Proposition 2. Let $t>0$ and let $M_{t}$ be the set function defined by formula (2) for the operators $S(t)=e^{i D t}, t \in \mathbb{R}$. Let $X_{s}, s \geq 0$, be the evaluation maps defined on the space $\Omega$ of all paths $\omega:[0, \infty) \rightarrow \mathbb{R}^{3}$. Then for any $0<t_{1}<t_{2}<t$, the set function $M_{t} \circ X_{\left\{t_{1}, t_{2}\right\}}^{-1}$ is unbounded on the algebra generated by all product sets $A \times B$, with $A, B \in \mathscr{B}\left(\mathbb{R}^{3}\right)$.

Proof. According to (2), $M_{t} \circ X_{\left\{t_{1}, t_{2}\right\}}^{-1}(A \times B)=S\left(t-t_{2}\right) Q(B) S\left(t_{2}-t_{1}\right) Q(A) S\left(t_{1}\right)$ for all $A, B \in \mathscr{B}\left(\mathbb{R}^{3}\right)$. It is enough to show the unboundedness of the collection of all operators $\sum_{j=1}^{k} Q\left(B_{j}\right) S\left(t_{2}-t_{1}\right) Q\left(A_{j}\right)$ with $A_{j} \times B_{j}, A_{j}, B_{j} \in \mathscr{B}\left(\mathbb{R}^{3}\right), j=$ $1, \ldots, k, k=1,2, \ldots$, a partition of $\mathbb{R}^{3} \times \mathbb{R}^{3}$. Suppose otherwise, that for each $u, v \in \mathbb{C}^{n}$ the set function $m: A \times B \mapsto\left(Q(B) S\left(t_{2}-t_{1}\right) Q(A) u, v\right)$ is actually the restriction to product sets of a bounded additive set function on the algebra generated by product sets. Then [J1, Proposition 2] shows that $m$ is the restriction of a Borel measure on $\mathbb{R}^{3} \times \mathbb{R}^{3}$, so for $T=S\left(t_{2}-t_{1}\right)$, the inequality (3) is certainly satisfied. By Theorem 1 , it follows that $S\left(t_{2}-t_{1}\right)$ is convolution with respect to a matrix valued measure.

However, if $S\left(t_{2}-t_{1}\right)$ were convolution with respect to a matrix valued measure $\mu$, then $S\left(t_{2}-t_{1}\right)$ would map the subspace $L^{2}\left(\mathbb{R}^{3}, \mathbb{C}^{4}\right) \cap L^{\infty}\left(\mathbb{R}^{3}, \mathbb{C}^{4}\right)$ of $L^{2}\left(\mathbb{R}^{3}, \mathbb{C}^{4}\right)$ into itself, continuously for the uniform norm. An elementary proof showing that this is not the case is given in [Z, II.1]. The operator $S\left(t_{2}-t_{1}\right)$ is actually convolution with respect a matrix valued distribution of order one, explicitly calculated in $[R]$. This contradiction shows that the original assumption that $M_{t} \circ X_{\left\{t_{1}, t_{2}\right\}}^{-1}$ is bounded must be false. The result is also a consequence of Theorem 2 below.

The matrices $\alpha_{1}, \alpha_{2}, \alpha_{3}$ in the Dirac operator do not commute. It turns out that this is the source of the unboundedness of the finite-dimensional distributions of the associated process.

Suppose that $A_{1}, \ldots, A_{d}$ are hermitian $n \times n$ matrices and $B$ is any $n \times n$ matrix. Then $H=\sum_{j=1}^{d} A_{j} \partial_{x_{j}}+B$ is the infinitesimal generator of a group $S$ of operators on $L^{2}\left(\mathbb{R}^{d}, \mathbb{C}^{n}\right)$. The operator $\partial_{x_{j}}$ is viewed as the generator of the group of translation operators in the $j$-th direction, acting on $L^{2}\left(\mathbb{R}^{d}, \mathbb{C}^{n}\right)$. 
Theorem 2. Let $t>0$ and let $M_{t}$ be the set function defined by formula (2) for the operators $S(t)=e^{i H t}, t \in \mathbb{R}$. Let $X_{s}, s \geq 0$, be the evaluation maps defined on the space $\Omega$ of all paths $\omega:[0, \infty) \rightarrow \mathbb{R}^{3}$. Then for some $0<t_{1}<t_{2}<t$, the set function $M_{t} \circ X_{\left\{t_{1}, t_{2}\right\}}^{-1}$ is bounded on the algebra generated by all product sets $A \times B, A, B \in \mathscr{B}\left(\mathbb{R}^{3}\right)$, if and only if the matrices $A_{1}, \ldots, A_{d}$ commute. If the matrices $A_{1}, \ldots, A_{d}$ do commute, then $M_{t}$ is actually bounded.

Proof. First suppose that $M_{t} \circ X_{\left\{t_{1}, t_{2}\right\}}^{-1}$ is bounded. Then as in the proof of Proposition 2, it follows from Theorem 1 that $S\left(t_{2}-t_{1}\right)$ is convolution with respect to a matrix valued measure $\mu$. Moreover, the spectral calculus for selfadjoint operators ensures that $S\left(t_{2}-t_{1}\right)$ is the Fourier multiplier operator $T_{\Phi}$ for the matrix valued function

$$
\Phi(\xi)=\exp \left(\left(i \sum_{j=1}^{d} A_{j} \xi_{j}+B\right)\left(t_{2}-t_{1}\right)\right), \quad \xi \in \mathbb{R}^{d} .
$$

By Proposition 1, $\Phi$ is the Fourier-Stieltjes transform of $\mu$.

According to [B, Lemma 1 (iii)], $\Phi$ belongs to the space of matrix multipliers on $L^{1}\left(\mathbb{R}^{d}, \mathbb{C}^{n}\right)$, and hence, by [B, Lemma 7], so does the function

$$
\xi \mapsto \exp \left(i \sum_{j=1}^{d} A_{j} \xi_{j}\left(t_{2}-t_{1}\right)\right), \quad \xi \in \mathbb{R}^{d} .
$$

An appeal to [B, Theorem 1] ensures that $A_{1}, \ldots, A_{d}$ commute.

If the matrices $A_{1}, \ldots, A_{d}$ commute, then we are in the situation considered in [I2] and [J1, Section 2], and the conclusion follows from the results there.

The point of Theorem 2 is that the boundedness of the finite-dimensional distributions of the process associated with a symmetric hyperbolic system already forces the system to be of a very restricted type.

\section{REFERENCES}

[B] P. Brenner, The Cauchy problem for symmetric hyperbolic systems in $L_{p}$, Math. Scand. 19 (1966), 27-37.

[D-U] J. Diestel and J. J. Uhl, Jr., Vector measures, Math. Surveys Monographs, vol. 15, Amer. Math. Soc., Providence, RI, 1977.

[G-J] J. Glimm and A. Jaffe, Quantum physics: A functional integral point of view, SpringerVerlag, New York, 1981.

[I1] T. Ichinose, Path integral for the Dirac equation in two space-time dimensions, Proc. Japan Acad. Ser. A Math. Sci. 58 (1982), 290-293.

[12] _ Path integral for a hyperbolic system of first order, Duke Math. J. 51 (1984), 1-36.

[J1] B. Jefferies, Processes associated with evolution equations, J. Funct. Anal. 91 (1990), 259-277.

[J2] - On the additivity of unbounded set functions, Bull. Austral. Math. Soc. 45 (1992), 223-236.

[J-O] B. Jefferies and S. Okada, Pettis integrals and singular integral operators, Illinois J. Math. 38 (1994).

[K] I. Kluvánek, Operator valued measures and perturbations of semi-groups, Arch. Rational Mech. Anal. 81 (1983), 161-180.

[R] G. Rosen, Feynman path summation for the Dirac equation: An underlying one-dimensional aspect of relativistic particle motion, Phys. Rev. A (3) 28 (1983), 1139-1140. 
[S] L. Schwartz, Radon measures in arbitrary topological spaces, Oxford Univ. Press., Tata Institute of Fundamental Research, Bombay, 1973.

[Si] B. Simon, Functional integration and quantum physics, Academic Press, New York, San Francisco, and London, 1979.

[Z] T. Zastawniak, Path integrals for the Dirac equation-some recent developments in the mathematical theory, Stochastic Analysis, Path Integration and Dynamics (K. D. Elworthy and J-C Zambrini, eds.), Pitman Res. Notes in Math., vol. 200, Longman Sci. Tech., Harlow, 1989, pp. 243-263.

School of Mathematics, UNSW, P.O. Box 1, Kensington, New South Wales 2032, AusTRALIA

E-mail address: B. Jefferies@unsw.edu.au 